\title{
The Exploration of BlockChain Technology in Academic Research Field
}

\author{
Juntao Zeng*, Fei Li, Hongwu Qin \\ College of Computer Science and Engineering, Northwest Normal University Lanzhou 730070, China \\ *Corresponding Author: 2018211730@nwnu.edu.cn
}

\begin{abstract}
The field of academic research is one of the hot reform fields at present, which has been widely concerned by many scholars and academic circles. As a new distributed infrastructure, blockchain technology has the characteristics of decentralization, point-to-point transmission, consensus mechanism and cryptography technology, and is especially suitable for academic research. In the academic field, the dissemination of research results, experimental "reproducibility", academic copyright and other issues. Combined with examples, this paper integrates blockchain with academic scenes, so as to improve the ecological environment of the current academic field and provide new ideas for the exploration of academic research field. Finally, this paper discusses the current research status and challenges of the academic application of block chain, and makes a prospect for the research.
\end{abstract}

Keywords: Blockchain, Dissemination of academic research results, Experimental "reproducibility", Academic copyright

\section{Introduction}

Since its birth in 2009, blockchain has been gradually applied to different fields after more than a decade of improvement and development. With the gradual clarity of blockchain concept, a large number of academic researchers have begun to pay attention to blockchain technology. Blockchain has great potential in the field of academic research due to its characteristics such as decentralization, transparency, traceability and non-tampering. Yang Xianmin, associate professor of Jiangsu Normal University, pointed out [1] that block chain technology will play an important role in the future education field, and it will build a new academic education resources and new ecology. Based on this, this paper briefly introduces the principle and core technology of block chain technology, analyzes the development and inspiration of block chain technology in various industries, and combines with the latest case analysis of academic research and application based on block chain technology to make a comprehensive induction and summary.

\subsection{The birth and development of Bitcoin}

At the end of 2008, Satoshi Nakamoto published A paper entitled Bitcoin:A Peer-to-peer Electronic Cash System in an anonymous and independent discussion group on cryptography[2]. In this paper, Satoshi Nakamoto envisions a new kind of virtual digital cryptocurrency, bitcoin, which uses a distributed ledger to store records and is fully decentralized (see Figure 1).
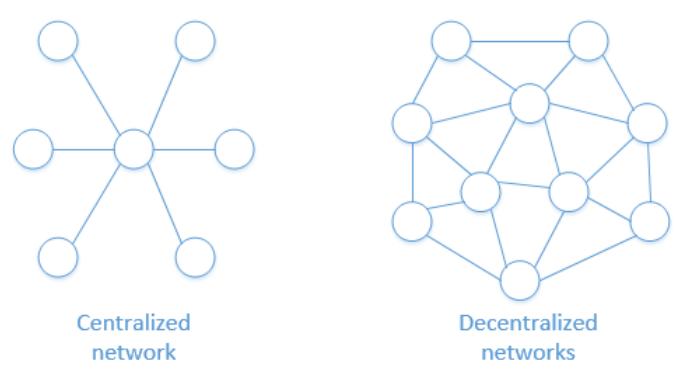

Figure 1 Decentralized networks and centralized networks

Published by Francis Academic Press, UK 


\subsection{Blockchain technology}

Blockchain technology is a new decentralized distributed ledger that integrates cryptography, consensus algorithm, timestamp and P2P transmission technology [3]. Each block contains a block header and a block body, where the block header includes the parent block hash, version number, timestamp, difficulty value, random number and Merkel root. The block body contains multiple transaction information. (See Figure 2).

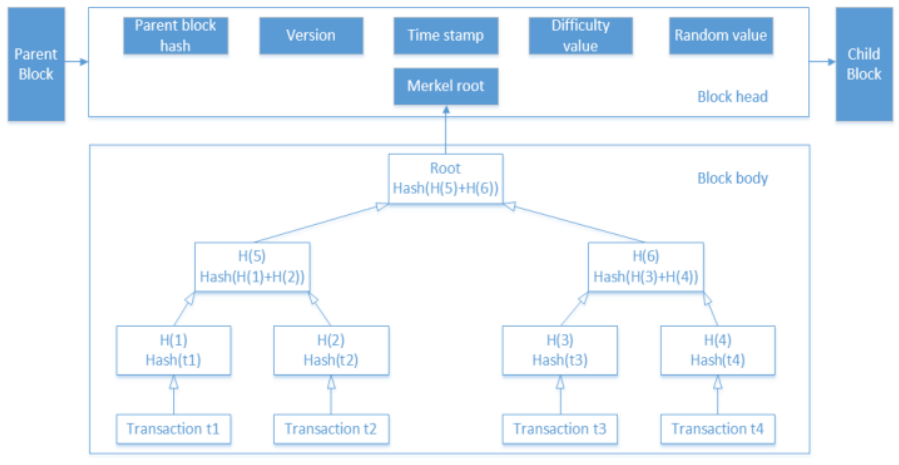

Figure 2 Block structure in the blockchain

\section{The Development of Blockchain in Various Industries}

The advantages of blockchain in various fields $[4,5,6,7,8,9]$ are shown in table 1 below.

Table 1 Advantages of blockchain in various industries

\begin{tabular}{|c|c|c|}
\hline Field & $\begin{array}{l}\text { Problems with traditional } \\
\text { methods }\end{array}$ & Based on the advantages of blockchain \\
\hline $\begin{array}{c}\text { The } \\
\text { medical } \\
\text { field }\end{array}$ & $\begin{array}{l}\text { 1) Centralized database } \\
\text { maintenance is difficult and } \\
\quad \text { costly. } \\
\text { 2) Vulnerable to malicious } \\
\text { external attacks. } \\
\text { 3) Easy to form "information } \\
\text { islands". }\end{array}$ & $\begin{array}{l}\text { 1) Asymmetric encryption. Effectively address the } \\
\text { security issues of medical information. } \\
\text { 2) Decentralize. Solve the "information island" } \\
\text { problem. } \\
\text { 3) Distributed ledger. Reduced the maintenance } \\
\text { cost of the hospital. }\end{array}$ \\
\hline $\begin{array}{c}\text { The } \\
\text { charitable } \\
\text { field }\end{array}$ & $\begin{array}{l}\text { 1) Management is chaotic and } \\
\text { inefficient. } \\
\text { 2) Trust issues. } \\
\text { 3) The charity process is not } \\
\text { transparent. }\end{array}$ & $\begin{array}{l}\text { 1) Untamperable. Ensure that charity data is } \\
\text { recorded on the blockchain and cannot be tampered } \\
\text { with. } \\
\text { 2) Transparency and traceability. The purpose of } \\
\text { each charitable donation is recorded on the chain, } \\
\text { making it possible for anyone, from anywhere, to } \\
\text { see where the money and goods are going. }\end{array}$ \\
\hline $\begin{array}{c}\text { Supply } \\
\text { chain } \\
\text { traceability } \\
\text { field }\end{array}$ & $\begin{array}{l}\text { 1) There is a serious information } \\
\text { gap between the suppliers. } \\
\text { 2) Data center management, easy } \\
\text { to tamper with. } \\
\text { 3) Has the very strong } \\
\text { centralization characteristic. }\end{array}$ & $\begin{array}{l}\text { 1) Transparency and traceability. Details of } \\
\text { everything from raw materials to goods are } \\
\text { recorded in the blockchain, allowing anyone to } \\
\text { track goods quickly, accurately and effectively. } \\
\text { 2) Untamperable. Block chain USES asymmetric } \\
\text { encryption, distributed storage and other methods } \\
\text { to ensure that data cannot be tampered with. }\end{array}$ \\
\hline $\begin{array}{l}\text { Certificate } \\
\text { and } \\
\text { copyright } \\
\text { fields }\end{array}$ & $\begin{array}{l}\text { 1) Traditional copyright } \\
\text { registration process cumbersome, } \\
\text { high cost. } \\
\text { 2) Copyright infringement is } \\
\text { difficult to find. } \\
\text { 3) The period of safeguarding } \\
\text { rights is long. }\end{array}$ & $\begin{array}{l}\text { 1) Decentralize, effectively simplify registration } \\
\text { process, reduce copyright protection cost. } \\
\text { 2) Open, break the island of copyright information, } \\
\text { promote the development of digital industry. } \\
\text { 3) Autonomy, through the intelligent contract, to } \\
\text { protect the copyright of the original author. }\end{array}$ \\
\hline
\end{tabular}




\section{The application of block chain in academic research field}

Through the survey found, chain blocks, as the representative of the emerging technologies at home and abroad academic researchers are trying to block chain technology is used to solve the disadvantages not only in academic field, in the hope of block chain technology will change the traditional academic communication environment, to create new, throughout the scientific research of the ecological system of academic exchange [10].

\subsection{Distributed records of scientific research data to speed up the dissemination of research results}

Distributed storage of scientific records of the whole process of scientific research is of great significance to the dissemination of scientific research results [11]. After many years of development, the academic field has formed a highly collaborative cycle. In this loop, researchers build on the work of other researchers to generate new ideas and conduct new studies. Therefore, how scientists disseminate their findings and achievements quickly becomes very important. As blockchain technology enters the 3.0 era, it will subvert the current publishing model $[12,13,14]$ and leave the initiative of science communication in the hands of scientists, so as to accelerate the speed of academic communication.

In order to remove the barriers to the dissemination of scientific knowledge, block chain technology is used to build a new academic exchange infrastructure, create an open, transparent and trustworthy distributed environment, and form a large sharing platform for academic research exchanges on a global scale.

\subsection{Put an end to academic misconduct and regain the "reproducibility" of experiments}

Scientific research is an important reflection of a country's innovation ability and scientific research level, and the publication of scientific research is to strengthen the academic exchanges between scholars from different regions, and to reflect the most cutting-edge technologies and ideas in the field of science to the public.

In the 21st century, mankind has entered the era of data-intensive scientific discovery, and the development of science and technology is achieved through the cooperation between people [15]. Many studies are based on the work of other researchers, which requires each researcher to be accountable for his or her own research results. In 2016, Nature conducted a survey on more than 1,500 researchers in a variety of fields, including chemistry, physics and environment. Two-thirds of the researchers said they had tried to replicate others' experiments but failed [16]. At present, the lack of reproducibility of experiments has become a general consensus in academic circles.

Pluto platform can prevent academic fraud through public free discussion, thus avoiding problems such as the opacity of the review process of papers and the possibility that reviewers may get wrong review opinions [17] (see Figure 3).

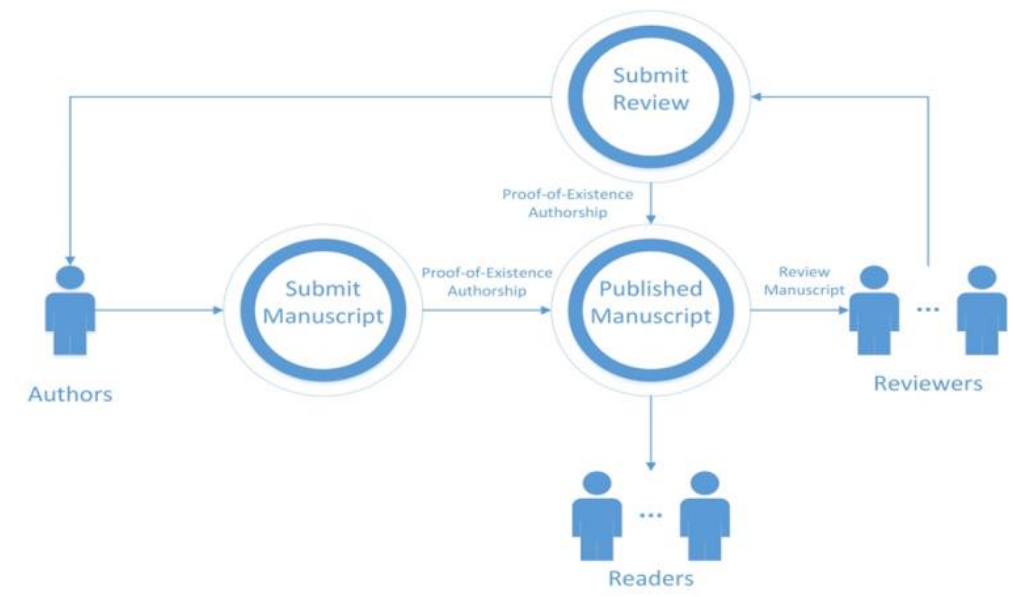

Figure 3. Public review 


\section{3 "Academic coin" micro payment to protect the original academic achievements}

Copyright protection has been a hot topic in recent years. With the advent of the digital age of the Internet, the digitization of labor achievements has become a trend. How to effectively protect the labor achievements of each original in this era of zero copy-paste cost has become an urgent problem to be solved.

The traditional copyright registration department has complicated procedures, long copyright confirmation cycle and high cost, which is not conducive to the copyright registration of the originator . The emergence of blockchain micro-payment mode will trigger a new revolution in the field of academic achievements copyright[18]. Its emergence will affect researchers, publishers and many consumers of academic resources. First, the publishers' monopoly would be broken. Secondly, the importance of journals as a platform for academic exchanges will be weakened. Finally, the value of a researcher's work is recognized, and the originator is paid.

On the academic exchange platform based on the block chain, the originators upload and publish their academic results, and independently set the corresponding price according to the value of the research results. Academic consumers need to use "Academic coin" to pay for viewing or downloading. If there are more than one originator, the "academic coin" can be split according to a preset proportion and transferred directly to each creator's wallet. This not only protects the academic achievements of the originator, but also increases the income of the originator and weakens the centrality of the publishing house.

By analyzing the drawbacks in the traditional academic research process and the new academic research platform based on block chain technology, this paper finds that block chain technology has many advantages in the academic research field, as shown in the following table:

Table 2 Advantages of blockchain in various academic research platforms

\begin{tabular}{ll}
\hline Platform & Based on the advantages of each academic research platform of block chain \\
\hline Orvium & 1) ORV platform records the entire scientific research life cycle, and makes the entire \\
scientific research process open and transparent on the block, so as to be traceable at any \\
time. \\
2) ORV platform can classify and organize scientific research according to scientific \\
research content. \\
3) ORV establishes an immutable, time-stamped, independent space for the proof of \\
author ownership for the experiment. \\
4) ORV allows authors to share unpublished data with other scientists to advance their \\
research. This gives researchers a new way of thinking that not only papers can be cited, \\
but research data and code can also be cited. \\
1) Pluto can record the experimental process and provide a way to solve the problem that \\
the experiment cannot be reproduced. \\
2) Pluto platform has a public free discussion module. \\
3) Pluto decentralized Platform USES block chain technology to break the barriers of \\
communication between academic circles and make academic communication efficient, \\
reasonable and transparent. \\
4) All rights to relevant works on Pluto platform are owned by the author himself. \\
1. DEC provides instant billing. \\
2. DEC provides copyright storage function.
\end{tabular}

\section{Research status and existing challenges}

Blockchain technology has been around for nearly 12 years. Compared with the financial field, the academic field is unique and complex. There is still a long way to go before the academic field can fully apply the block chain technology. From the point of view of the present stage, the main findings are facing the following problems.

First of all, privacy is still the most difficult problem to solve in the current blockchain application. When users are not willing to disclose their own information and information, how to ensure that user information is not disclosed and how to protect the privacy of user information [19].

Secondly, how to guarantee efficiency and safety? In the real world, efficiency and security are often in conflict. While ensuring the security of data link with high efficiency is also an issue that must 
be considered, which is extremely challenging at present [20].

Finally, the current public chain has the characteristics of non - tampering and non - deleting. If users modify relevant data, they must upload new complete data, which greatly aggravates the burden of distributed storage [21].

\section{Prospect}

In the current blockchain 3.0 era, blockchain is expected to play a more important role in different fields. As a new technology, block chain is still in the dynamic development process. From the perspective of human social development, the ultimate form of human society is the trusting autonomous society [21], which is exactly in line with the vision in the academic research process. In the new academic research ecosystem in the future, academic research will be more rigorous and more in line with academic rules.

\section{References}

[1] Yang Xianmin,Li Xin,Wu Huanqing,Zhao Keyun.The Application Model and Challenges of Blockchain Technology in Education,2017(02):34-45.

[2] Bitcoin:a peer-to-peer electronic cash system[Online]. Nakamoto S. https://bitcoin.org/bitcoin.pdf . 2009

[3] Shao Qi-Feng,Jin Che-Qing,Zhang Zhao,Qian Wei-Ning,Zhou Ao-Ying.Blockchain:Architecture and Research Progress, 2018,41

[4] Xiao Yue, Huiju Wang, Dawei Jin. Healthcare Data Gateways: Found Healthcare Intelligence on Blockchain with Novel Privacy Risk Control[J]. Journal of Medical Systems, 2016, 40(10).

[5] Mettler M. Blockchain technology in healthcare: The revolution starts here[C]// 2016 IEEE 18th International Conference on e-Health Networking, Applications and Services (Healthcom). IEEE, 2016. [6] Farooq Muhammad Shoaib,Khan Misbah,Abid Adnan. A framework to make charity collection transparent and auditable using blockchain technology[J]. Computers and Electrical Engineering,2019,83.

[7] R. Kumar and R. Tripathi, "Traceability of counterfeit medicine supply chain through Blockchain," 2019 11th International Conference on Communication Systems \& Networks (COMSNETS), Bengaluru, India, 2019, pp. 568-570, doi: 10.1109/COMSNETS.2019.8711418.

[8] R. C. Basole, M. A. Bellamy, H. Park and J. Putrevu, "Computational Analysis and Visualization of Global Supply Network Risks," in IEEE Transactions on Industrial Informatics, vol. 12, no. 3, pp. 1206-1213, June 2016, doi: 10.1109/TII.2016.2549268.

[9] Jnoub N, Klas W. Detection of Tampered Images Using Blockchain Technology[C]// International Conference on Blockchain and Cryptocurrency. Multimedia Information System, University of Vienna, Währinger Str. 29, 1090, Austria;Multimedia Information System, University of Vienna, Währinger Str. 29, 1090, Austria; 2019.

[10] Y. Zou, T. Meng, P. Zhang, W. Zhang and H. Li, "Focus on Blockchain: A Comprehensive Survey on Academic and Application," in IEEE Access, vol. 8, pp. 187182-187201, 2020, doi: 10.1109/ACCESS.2020.3030491.

[11] Y. Xu, "Section-Blockchain: A Storage Reduced Blockchain Protocol, the Foundation of an Autotrophic Decentralized Storage Architecture," 2018 23rd International Conference on Engineering of Complex Computer Systems (ICECCS), Melbourne, VIC, 2018, pp. 115-125, doi: 10.1109/ICECCS2018.2018.00020.

[12] G. Bu, T. S. L. Nguyen, M. Potop Butucaru and K. L. Thai, "HyperPubSub: Blockchain Based Publish/Subscribe," 2019 38th Symposium on Reliable Distributed Systems (SRDS), Lyon, France, 2019, pp. 366-3662, doi: 10.1109/SRDS47363.2019.00052.

[13] S. R. Niya et al., "A Blockchain-based Scientific Publishing Platform," 2019 IEEE International Conference on Blockchain and Cryptocurrency (ICBC), Seoul, Korea (South), 2019, pp. 329-336, doi: 10.1109/BLOC.2019.8751379.

[14] J. Putsorn, S. Nontree and T. Chomsiri, "JS Digital Assets Trading System," 2019 Joint International Conference on Digital Arts, Media and Technology with ECTI Northern Section Conference on Electrical, Electronics, Computer and Telecommunications Engineering (ECTI DAMTNCON), Nan, Thailand, 2019, pp. 13-16, doi: 10.1109/ECTI-NCON.2019.8692301.

[15] Hara N, Solomon P, Kim S L, et al. An emerging view of scientific collaboration: Scientists' perspectives on collaboration and factors that impact collaboration[J]. Journal of the Association for 
Information ence \& Technology, 2014, 54(10):952-965.

[16] A. Adorjan and I. F. de Kereki, "Academic misconduct in projects: Perspective of students and teachers of Introductory Computer Science courses, " 2017 XLIII Latin American Computer Conference (CLEI), Cordoba, 2017, pp. 1-7, doi: 10.1109/CLEI.2017.8226375.

[17] Y. Liping, P. Yuntao, Y. Chun and W. Yishan, "Study on Peer Review and Multi-indicators Evaluation in Scientific and Technological Assessment," 2008 International Symposium on Knowledge Acquisition and Modeling, Wuhan, 2008, pp. 794-798, doi: 10.1109/KAM.2008.39.

[18] Q. Huang and Y. Zhao, "A Secure and Lightweight Micro-Payment Scheme in P2P Networks," 2009 International Conference on Industrial and Information Systems, Haikou, 2009, pp. 134-137, doi: 10.1109/IIS.2009.64.

[19] R. Henry, A. Herzberg and A. Kate, "Blockchain Access Privacy: Challenges and Directions," in IEEE Security \& Privacy, vol. 16, no. 4, pp. 38-45, July/August 2018, doi: 10.1109/MSP.2018.3111245. [20] G. Sun, M. Dai, J. Sun and H. Yu, "Voting-based Decentralized Consensus Design for Improving the Efficiency and Security of Consortium Blockchain," in IEEE Internet of Things Journal, doi: 10.1109/JIOT.2020.3029781.

[21] YUAN Yong, WANG Fei-Yue.Blockchain: The State of the Art and Future Trends,2016,42 (04):481-494. 